\title{
Curcumin Alleviates Chronic Pain and Improves Cognitive Impairment via Enhancing Hippocampal Neurogenesis in Sciatic Nerve Constriction Rats
}

\author{
Jingyi Du* \\ Yifan Deng* \\ Zhuolin Qiu \\ Guoliang Sun \\ Yue Guo \\ Ziqing Hei \\ Xiang Li $(\mathbb{D}$
}

Department of Anesthesiology, The Third Affiliated Hospital, Sun Yat-Sen University, Guangzhou, People's Republic of China

*These authors contributed equally to this work
Correspondence: Xiang Li

Department of Anesthesiology, The Third Affiliated Hospital of Sun Yat-sen

University, Number 600, Tianhe Road,

Guangzhou, 510630, People's Republic of

China Tel/Fax +86208525 3132

Email lixiang27@mail.sysu.edu.cn
Purpose: Cognitive impairment is a complication that most frequently happens in patients with chronic neuropathic pain and has limited effective therapy. The aim of this study was to explore the effects of curcumin on the cognitive deficit in rats with peripheral nerve injury induced-neuropathic pain.

Methods: The neuropathic pain rat model was constructed using chronic constriction injury (CCI). The curcumin $(60 \mathrm{mg} / \mathrm{kg})$ or vehicle was intraperitoneally administered once a day, beginning at 14 th day after surgery and continued for 14 consecutive days. The nociceptive threshold tests were measured by paw mechanical withdraw threshold (PMWT) and paw thermal withdrawal latency (PTWL), while the spatial memory abilities were evaluated by the Morris water maze test. The mean counts of bromodeoxyuridine (Brdu)/neuronal nuclei (NeuN) as well as Brdu/doublecortin (DCX) co-labeled cells were used to evaluate neurogenesis in the dentate gyrus of hippocampus. The ultrastructure of the synapse in hippocampal region was visualized using transmission electron microscopy (TEM).

Results: Increased PMWT and PTWL, as well as relieved memory deficits, were found in CCI rats under curcumin administration. Moreover, curcumin treatment increased the number of newly born immature $(\mathrm{BrdU} / \mathrm{NeuN})$ and newly generated mature neurons (BrdU/ DCX). The TEM examination revealed increased PSD thickness and shorter active zone length as well as narrowed synaptic cleft width in the hippocampal region of CCI rats after curcumin injection.

Conclusion: Curcumin can alleviate CCI induced nociceptive behaviors and memory deficit. This effect might be associated with hippocampal neurogenesis and synaptic plasticity improvements, which indicated curcumin as a potential strategy for the cognitive impairment restoration under prolonged neuropathic pain condition.

Keywords: curcumin, peripheral nerve injury, cognitive impairment, hippocampus neurogenesis

\section{Introduction}

Patients with chronic pain frequently experienced cognitive impairment commonly characterized as memory and learning capacity deterioration, which deteriorates the quality of life and even increases patients' financial burdens. ${ }^{1}$ Numerous evidences have revealed that memory deficits in patients with chronic pain could be attributed to the severity of the patients' emotional disturbances, including anxiety and depression. $^{2,3}$ Previous studies report the mean prevalence rates of around $20-30 \%$ for cognitive function impairment in patients with chronic neuropathic 
pain. ${ }^{4,5}$ Preclinical researches revealed that the neuropathic pain-related behaviors usually accompany with spatial learning as well as memory disorders in murine models with chronic peripheral nerve injury. ${ }^{6,7}$ However, although the link between chronic pain and the development of cognitive dysfunction has been extensively studied and documented, limited therapeutic options are available up to now, indicating that the molecular aspects underlying chronic pain-associated learning and memory deficits have not been well understood.

The hippocampus, a crucial component of the limbic system, is considered to play an important role during memory and mood formation in brain. Recent preclinical evidences have showed that chronic peripheral nerve injury exposure could decrease neurogenesis process in the dentate gyrus (DG) granular cell layer of adult hippocampus. ${ }^{1,8}$ The deficits in hippocampal neurogenesis might decline the generation of new immature neurons in hippocampus that promote the clearance of panic memory and maintain the ability of animal to cope with environmental challengers, ${ }^{9}$ while restoring the impaired hippocampal neurogenesis in chronic neuropathic or inflammatory pain models were beneficial for alleviation of painful syndromes. ${ }^{10}$ Additionally, it is noteworthy that the animals with a reversible neuropathic injury might persistently display cognitive impairment behaviors accompanied with constant ablation of hippocampal neurogenesis, indicating even after recovery from the physical pain of the neuropathy, its more long-term stressing consequences endure on the nervous system, and that impaired neurogenesis might be involved in the emergence of these consequences. ${ }^{11}$ Thus, enhancing the blocked hippocampal neurogenesis after peripheral nerve injury might be a key to improve cognitive function during chronic pain.

Curcumin, a phenolic compound derived from the rhizome of Curcuma longa, has been widely studied for its analgesic properties against various neuropathic pains. $^{12-15}$ Recently, curcumin was demonstrated to be able to improve pain behaviors as well as cognitive impairments in cobra venom-induced trigeminal neuralgia and chronic constriction injury (CCI) rodents, which suggested the benefit effects of curcumin on neuroprotective property and improvement of learning and memory performances in chronic neuropathic pain models. ${ }^{7,16}$ However, it remains unclear whether curcumin treatment can increase hippocampal neurogenesis and improve cognitive dysfunctions in neuropathic pain elicited by peripheral nerve injury.
This study was designed to report a causal evidence that curcumin plays a potential role in improving cognitive function in chronic constriction injury (CCI) pain model owing to its hippocampal neurogenesis-promoting property.

\section{Methods}

\section{Study Approval and Animal Preparation}

All protocols of the current study were approved by the Animal Ethical Committee of the Third Affiliated Hospital, Sun Yat-Sen University, and conducted in accordance with the guidelines of the International Association for the Study of Pain.

Male Sprague Dawley rats (200-250 g) were obtained from the Experiment Animal Center of Guangdong Province (SCXK [Yue] 20180002) and maintained in separate cages under a 12-h light/dark cycle, with water and fed available ad libitum. The room was kept at temperature $\left(23 \pm 2^{\circ} \mathrm{C}\right)$ as well as humidity $(50-60 \%)$. We made all efforts to minimize the number of animals used and their suffering.

\section{Procedures of Pain Model Establishment}

Anesthesia was administrated by intraperitoneal injection of pentobarbital sodium $(50 \mathrm{mg} / \mathrm{kg})$. Then, rats were performed either a unilateral CCI or a sham procedure as depicted by Bennett et al. ${ }^{17}$ In short, the CCI model was established by a ligation at the left sciatic nerve using four ligatures with non-absorbable $4 / 0$ silk threads (1.0- to 1.5-mm spacing between each ligature), while only exposure of the left sciatic nerve without ligation was performed on sham rats.

\section{Drug Treatments}

By a random table, 40 rats were randomly divided into four groups, as follows (i) sham with curcumin group (Sham + Cur, $\mathrm{n}=10$ ); (ii) CCI with curcumin group (CCI + Cur, n = 10), (iii) sham with vehicle group (Sham + Veh, $\mathrm{n}=10$ ), or (iv) CCI with vehicle group (CCI + Veh, $\mathrm{n}=10$ ). According to the study from Zhu et al, ${ }^{11}$ curcumin (Sigma-Aldrich, Santa Clara, CA, USA) was dissolved in $20 \%$ dimethyl sulfoxide (DMSO) with $80 \%$ normal saline solution. The curcumin $(60 \mathrm{mg} / \mathrm{kg})$ or vehicle (20\% DMSO with $80 \%$ normal saline solution) was intraperitoneally administered once a day, which start at 14th day after surgery and continued for 14 consecutive days (Figure 1). 


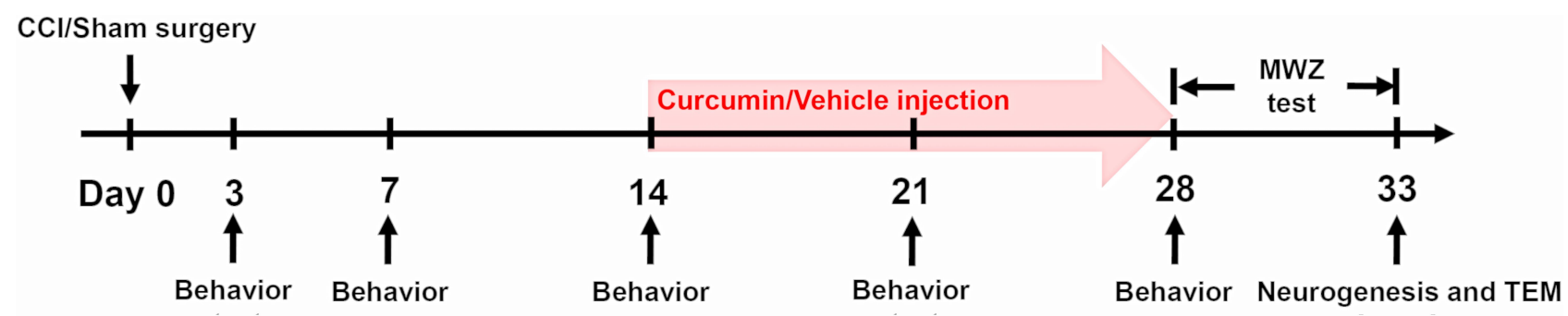

Figure I Timeline of $\mathrm{CCl}$ or sham surgery, curcumin or vehicle (20\% DMSO with $80 \%$ normal saline solution) injection, behavior and MWZ tests, neurogenesis and TEM detection.

Abbreviations: $\mathrm{CCl}$, chronic constriction injury; DMSO, dimethyl sulfoxide; MWZ, Morris water maze; TEM, transmission electron microscopy.

\section{Mechanical and Thermal Nociceptive Threshold Test}

In order to determine whether the CCI model was well established, both paw mechanical withdraw threshold (PMWT) and paw thermal withdrawal latency (PTWL) were assessed 3 days before surgery and 3, 7, 14, 21 and 28 days by two research staffs who were blinded to the group (Figure 1). The behavior test was performed in a noise-free room. Each rat was placed individually on a wire mesh grid (mechanical nociceptive threshold test) or glass floored box (thermal nociceptive threshold test). At least $30 \mathrm{~min}$ was allowed to habituate to the surroundings.

The PMWT was assessed by the von Frey monofilaments (North Coast Medical, California, USA) weighing $1.0-15.0 \mathrm{~g}$. The ascending series monofilaments were used to perpendicularly stimulate the mid-plantar surface of hind paw, and each was presented five times with an interval of 4-6 s. A positive response was considered a withdrawal, along with shaking or licking of the paw at least three times over the course of five applications. The smallest bending force of the monofilament eliciting a positive response was determined to be the PMWT.

The PTWL was measured through the thermal test apparatus (UGO BASILE 37370 Plantar Test Apparatus, Italy), which could release a focused radiant heat source from below the glass onto the mid-plantar surface of the hind paw. According to Dai et al, ${ }^{18}$ the infrared intensity was set as 45 with a cutoff time of $25 \mathrm{~s}$ to avoid cutaneous damage to the hind paw. This thermal nociceptive threshold test was repeated five times, and the PTWL was considered as the mean latency of withdrawal response across five tests.

\section{Morris Water Maze Test}

When the curcumin had been administrated for 14 consecutive days, a five-day Morris water maze (MWZ) test was applied to evaluate the spatial memory abilities, according to previous $\operatorname{study}^{19}$ (Figure 1). Briefly, a circular pool $(180 \mathrm{~cm}$ diameter and $60 \mathrm{~cm} \mathrm{high})$, which was full of $19^{\circ} \mathrm{C}$ to $22^{\circ} \mathrm{C}$ opaque water and divided into four equivalent quadrants $(\mathrm{N}, \mathrm{E}, \mathrm{S}$, and $\mathrm{W})$, was prepared for the MWZ test. A hidden escape platform was placed in one of four quadrants and submerged $0.5 \mathrm{~cm}$ below the water. For the first four days, rodents underwent four acquisition trials separated by 20 -minute inter-trial interval per day. During the four acquisition trials, rats were placed into the water facing the pool at different predetermined positions. The swimming speed and escape latency were recorded. On fifth day, the hidden escape platform was removed, and rats were placed into the pool at the location opposite to the platform position and allowed to swim freely for 120 s. According to the previous study, ${ }^{20}$ we recorded the percentage time spent in the target quadrant and times across platform. Moreover, the time spent in the platform quadrant and the remaining three quadrants were respectively recorded for each group. Whole MWZ test was performed by two research staffs who were blinded to group allocation.

\section{BrdU Administration}

To examine the effects of curcumin on neural precursor cells proliferation in the DG of the hippocampus, rats received intraperitoneal injection of $100 \mathrm{mg} / \mathrm{kg}$ bromodeoxyuridine (BrdU) (Sigma-Aldrich, B5002) once a week studying from the day receiving CCI or sham surgery until sacrifice, which was according to the previous study. ${ }^{10}$ Two hours after the last BrdU injection, rats were anesthetized and hippocampus tissues were prepared for the following immunofluorescent and transmission electron microscopy. 


\section{Immunofluorescent}

The hippocampus tissues were separated after MWZ test (Figure 1) and post-fixed at $4{ }^{\circ} \mathrm{C}$ overnight, followed by the dip in a $30 \%$ sucrose solution for $48 \mathrm{~h}$. Sections of $30 \mu \mathrm{m}$ thick were prepared by a freezing microtome (VT1000S, Leica Microsystems). For all cases, slices were incubated with the mouse anti-doublecortin (DCX, 1:500, ab254133, Abcam), rabbit anti-BrdU (1:200, ab152095, Abcam), mouse antineuronal nuclei (NeuN, 1:400, ab104224, Abcam) antibodies. The secondary antibodies were tagged with goat anti-mouse Alexa 594 (1:500, ab150120, Abcam), goat anti-rabbit Alexa 488 (1:500, ab150081, Abcam). Then slices were stained with 4',6-diamidino-2-phenylindole (DAPI, $0.5 \mu \mathrm{g} / \mathrm{mL}, 4083 \mathrm{~s}$, Cell Signaling Technology). After staining, slices were visualized by two experimenters blinded to group allocation with the laser scanning confocal microscopy (FV1000; Olympus). The mean counts of Brdu/NeuN as well as Brdu/DCX colabeled cells in every fifth section for each animal was used to evaluate neurogenesis in the DG.

\section{Transmission Electron Microscopy Examination}

The collected hippocampus tissues from rats were perfused in 2\% paraformaldehyde and 2\% glutaraldehyde in $0.1 \mathrm{M}$ sodium cacodylate, followed by post-fixation in $2 \%$ osmium tetroxide with $1.6 \%$ potassium ferrocyanide in 0.1 M sodium cacodylate. Subsequently, the hippocampus tissue samples were cut into small clumps of a volume of $1 \mathrm{~mm}^{3}$ and dehydrated through a graded acetone series, after which they were embedded in Eponate 812 medium (90529-77-4, Structure Probe, Inc., Pennsylvania, USA). Finally, the sections were placed on copper slot grids and stained with $2 \%$ uranyl acetate and lead citrate. Images were visualized by Hitachi HT-7700 transmission electron microscopy (Tokyo, Japan). As previously described, ${ }^{21,22}$ the morphologic features of synapse including postsynaptic dense (PSD) band, active zone and synaptic cleft were observed, and the mean values of thickness of the postsynaptic density, length of active zone as well as width of the synaptic cleft in every fifth DG region section was calculated.

\section{Statistical Analysis}

Data analysis and statistics were performed by SPSS 20.0 software (IBM CORP, New York, USA). Data were presented as mean \pm standard deviation (SD), and analyzed by a two-way analysis of variance (ANOVA) with repeated measures followed by Bonferroni post hoc comparisons. Statistical significance was set as $\mathrm{P}<0.05$.

\section{Results}

\section{Effect of Curcumin on Mechanical} Allodynia and Thermal Hyperalgesia

In comparison to rats in the Sham + Cur and Sham +Veh groups, those in the $\mathrm{CCI}+$ Veh group exhibited significant reductions of the mechanical threshold $(P<0.001$; Figure 2A) and thermal latency $(P<0.001$; Figure 2B) after sciatic nerve injury, which were throughout the 28day observation period. However, curcumin treatment markedly attenuated the mechanical allodynia $(P<$ 0.001 ; Figure $2 \mathrm{~A})$ and thermal hyperalgesia $(P<0.001$; Figure $2 \mathrm{~B}$ ) in rats from $\mathrm{CCI}+\mathrm{Cur}$ group. These results suggested that consecutively administrating $60 \mathrm{mg} / \mathrm{kg}$ curcumin for 14 days might alleviate both mechanical and thermal hyperalgesia resulted from peripheral nerve injury.

\section{Effect of Curcumin on Spatial Memory Function}

Compared to the sham rats received vehicle, the significantly longer escape latency to platform $(P<0.001$, Figure $3 \mathrm{~A}$ ) for $\mathrm{CCI}$ rats treated with vehicle indicated that the cognitive impairment was induced by peripheral nerve injury. While the administration of curcumin restored the increased escape latency resulted from CCI $(P<0.001$, Figure 3A). However, we did not observe any significant differences in swimming speed among four groups (Figure 3B). The rats in CCI-Veh group presented significant shorter swimming time in target quadrant $(P<0.001$, Figure 3C) and time across platform was reduced $(P<$ 0.001 , Figure $3 \mathrm{C})$ than those for the rats from sham-Veh group; on the contrary, both parameters were significantly prolonged after the injection of curcumin $(P=0.021$ for time in target quadrant, $P=0.019$ for time across platform, Figure $3 \mathrm{C}$ and $\mathrm{D}$ ). Besides, there was no significant difference in the spent time among four quadrants in CCI rats treated with vehicle, while the CCI rats treated with curcumin spent more time in the platform quadrant than other three quadrants $(P<0.01$, Figure 4$)$.

\section{Effect of Curcumin on Hippocampal Neurogenesis in DG Region}

Double labeling of proliferating neurons with anti-BrdU and other marker could provide indication for the phenotype of marked cells. ${ }^{23}$ Thus, we counted the number of double 
A

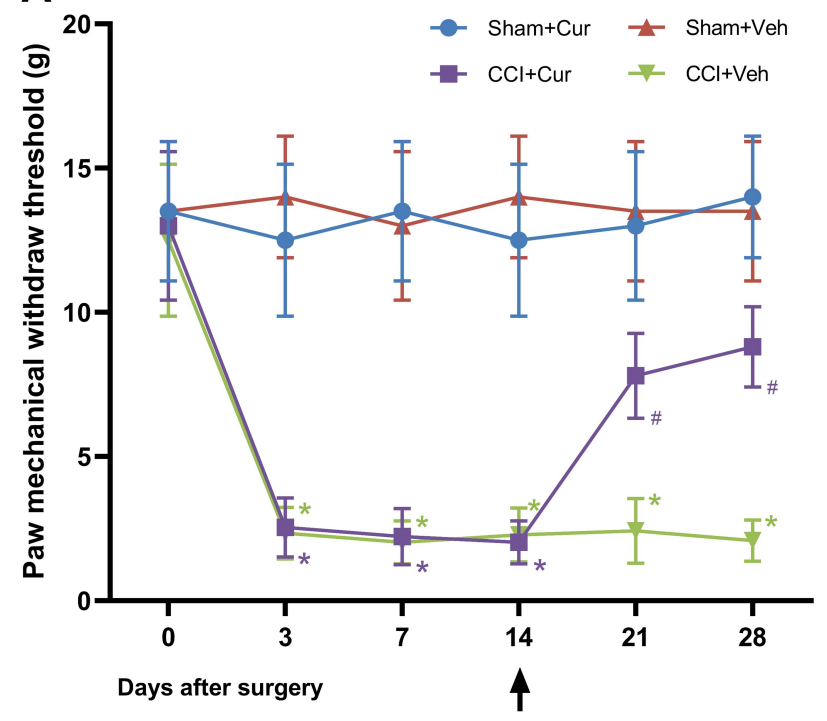

Start of curcumin injection

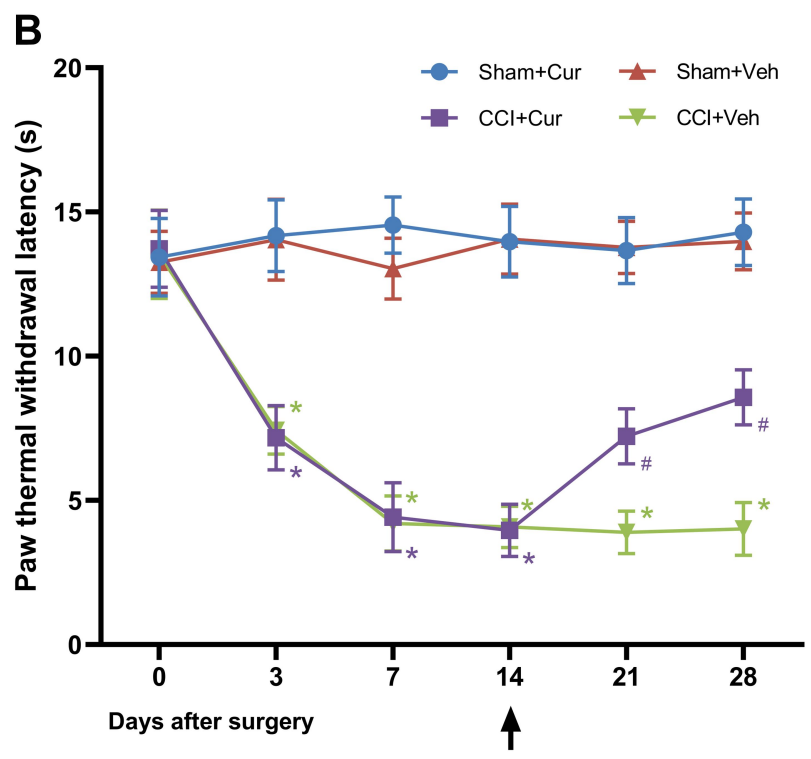

Start of curcumin injection

Figure 2 Time-course changes of mechanical threshold $(\mathbf{A})$ and thermal latency $(\mathbf{B})$ in the ipsilateral hind-paw of rats. Note: $* \mathrm{P}<0.001$ vs Sham+Veh, \#P $<0.001$ vs $\mathrm{CCl}+\mathrm{Veh}$.

Abbreviations: $\mathrm{CCl}$, chronic constriction injury; Veh, vehicle; Cur, curcumin.

immunostaining cells to identify newly born immature $(\mathrm{BrdU} / \mathrm{NeuN})^{24}$ and newly generated mature neurons (BrdU/DCX), ${ }^{23}$ respectively.

Four weeks after injury, the number of BrdU/NeuN (Figure 5) and BrdU/DCX (Figure 6) neurons was significantly lower in the dentate gyrus of the rats in CCI-Veh group compared to those in sham-Veh group Sham mice $(P=0.041$ for BrdU/NeuN, $P<0.001$ for BrdU/DCX). Notably, the number of BrdU/NeuN and BrdU/DCX double labeled neurons in the dentate gyrus was dramatically increased in the curcumin administered CCI rats $(P=0.014$ for $\mathrm{BrdU} / \mathrm{NeuN}$, $P=0.015$ for BrdU/DCX, Figures 5 and 6).

\section{Effect of Curcumin on the Hippocampal DG Synapse Density}

Compared with the Sham-Veh groups, a noticeably changed synapse density along with the lesser PSD thickness $(P=0.002)$, shorter active zone length $(P=0.006)$ and wider synaptic cleft width $(P=0.001)$ was found in the DG region of the CCI-Veh group (Figure 7). In contrast, when the CCI rats were treated with curcumin, the aforementioned abnormality of hippocampal synapse density in CCI-Veh rats appeared to return to be regular $(P=0.036$ for PSD thickness, $P=0.044$ for active zone length, $P=0.038$ for synaptic cleft width), similar to the morphologic features of synapse in sham rats (Figure 7).

\section{Discussion}

Our current results make the argument that curcumin treatment alleviated pain and improved cognition deficits in a peripheral nerve injury-induced neuropathic pain rat model. Furthermore, the protective effect of curcumin against neuropathic pain-caused cognitive impairment is partly associated with improving hippocampal neurogenesis.

Various functional and morphological alterations that related to the spatial learning and memory function impairments had been studied in neuropathic pain murine models. For example, Saffarpour $S$ et al found that the increase in GABA concentration and decrease in the glutamate and BDNF levels in the CA1 region of the hippocampus were associated with spatial learning and memory function disorders comorbid in CCI rats. ${ }^{25}$ Our current study using the CCI model shows that a prolonged pain performance (28day CCI) impairs spatial memory formation along with reductions in the numbers of newly born immature and newly generated mature neurons in hippocampal DG region, which were in accordance with prior studies. ${ }^{1,10}$ It was demonstrated that newborn neurons in the DG region are incorporated into the hippocampal network in existence and contributed to new cellular and behavioral capacities. $^{26,27}$ Compared with their mature counterparts, newborn neurons in the DG region were appeared to be more involved in new experience-related learning capacity 
A

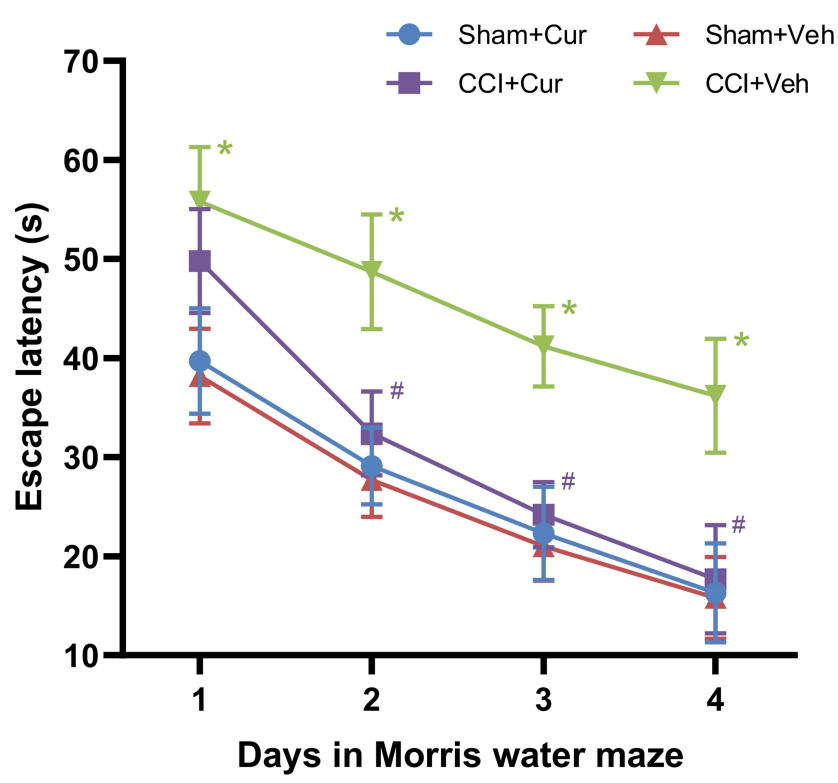

C

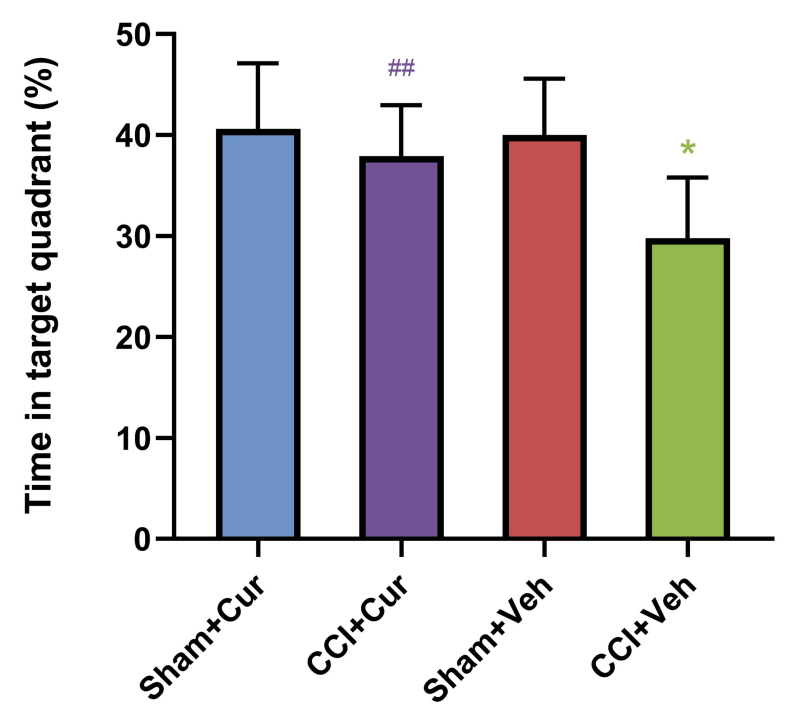

B

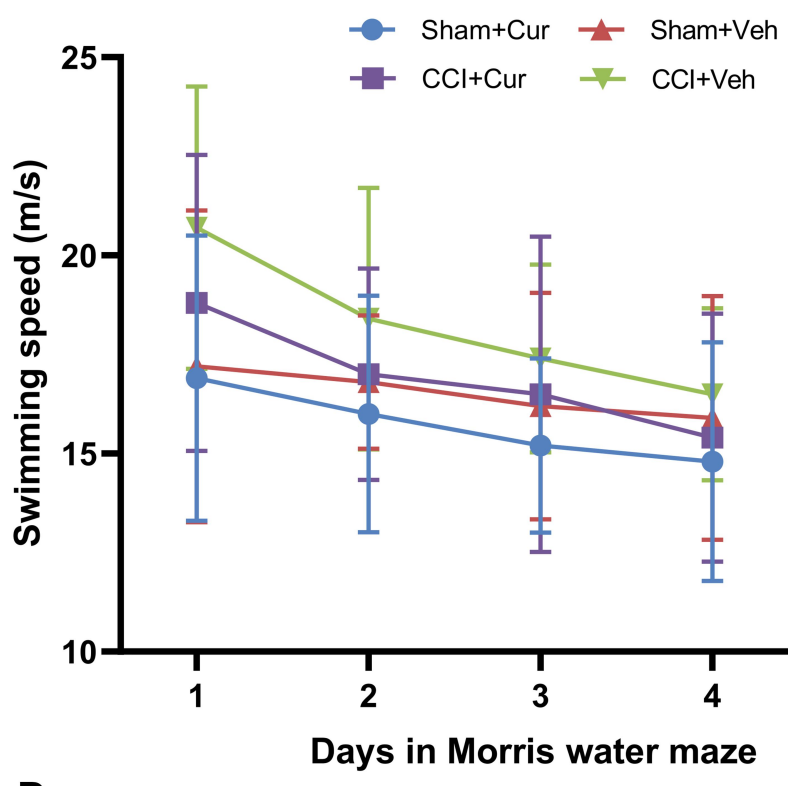

D

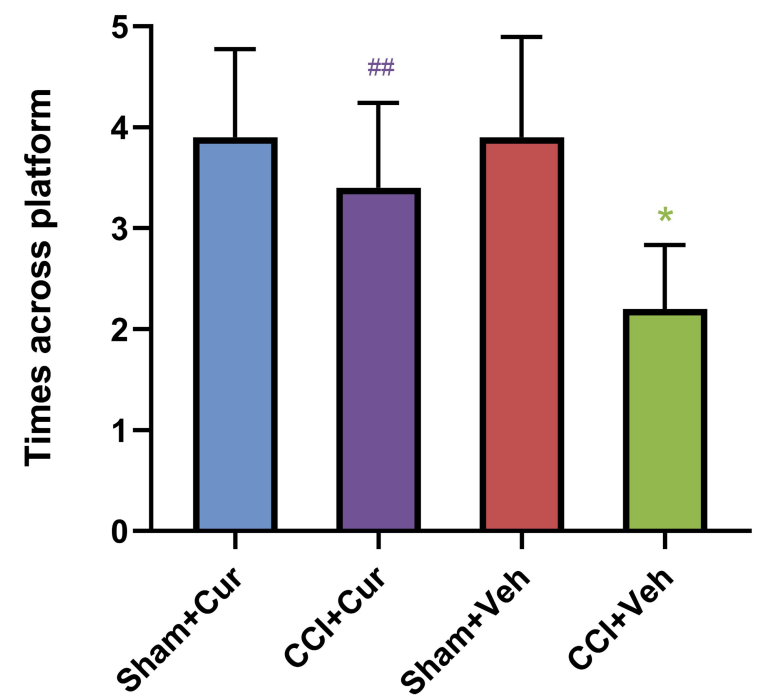

Figure 3 Time-course changes of escape latency (A), swimming speed (B), swimming time in target quadrant (C), time across platform (D) and spending times in four quadrants in the rats from different groups.

Note: $* \mathrm{P}<0.001$ vs Sham + Veh, \#P $<0.001$ vs $\mathrm{CCl}+\mathrm{Veh}$, \# $\mathrm{P}<0.05$ vs $\mathrm{CCl}+\mathrm{Veh}$.

Abbreviations: $\mathrm{CCl}$, chronic constriction injury; Veh, vehicle; Cur, curcumin.

and memory formation. ${ }^{28}$ More specifically, mice received mitotic inhibitor displayed increased escape latency and decreased time spent in the target quadrant, accompanied by a reduced number of newborn neurons in the DG region. ${ }^{1}$ These results confirm that disruption of hippocampal neurogenesis may at least in part act as a trigger to spatial memory impairment during the development of neuropathic pain.

Considering the connection between hippocampal neurogenesis and memory formation, it was hypothesized that facilitating hippocampal neurogenesis would be helpful to improve integration of newborn neurons into hippocampal networks and alleviate pain-induced spatial memory impairment. Our results revealed that repeated administration of $60-\mathrm{mg} / \mathrm{kg}$ curcumin was able to relieve spatial memory impairment as well as nociceptive behavior in CCI rats, which were in concert with the previous studies. ${ }^{12-16}$ More specifically, such effects of curcumin on cognitive impairments were appeared to be associated with improvement of hippocampal neurogenesis in CCI rats. The earlier findings 


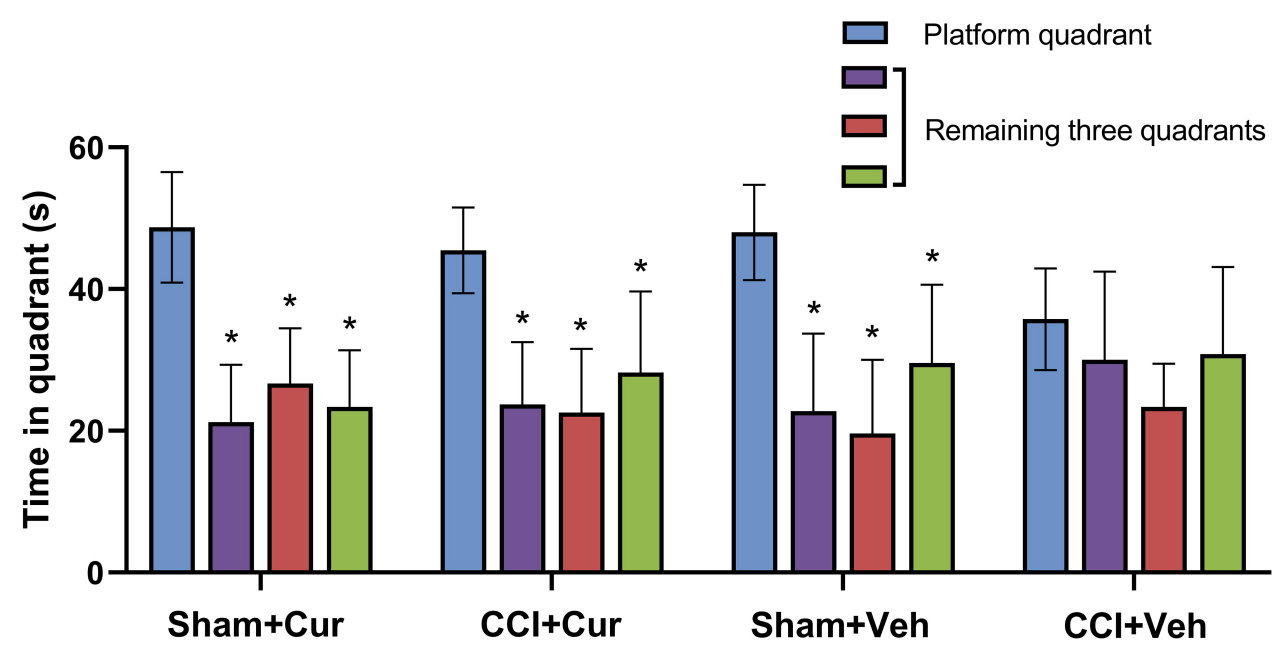

Figure 4 Time spent in four quadrants in the rats from different groups.

Note: $* \mathrm{P}<0.001$ vs time spent in platform quadrant.

Abbreviations: $\mathrm{CCl}$, chronic constriction injury; Veh, vehicle; Cur, curcumin
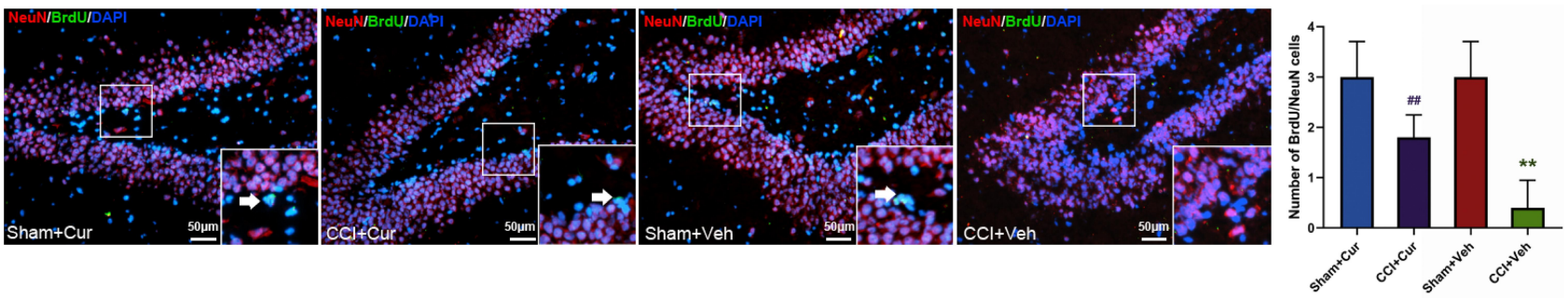

Figure 5 Representative images and quantitative summary of hippocampal NeuN/BrdU cells in the DG regions from rats in different groups (The white arrows represent NeuN/BrdU double labeled neurons).

Note: $* * \mathrm{P}<0.05$ vs Sham+Veh, \# $\mathrm{P}<0.05$ vs $\mathrm{CCl}+\mathrm{Veh}$.

Abbreviations: $\mathrm{CCl}$, chronic constriction injury; Veh, vehicle; Cur, curcumin; BrdU, bromodeoxyuridine; NeuN, neuronal nuclei; DAPI, 4',6-diamidino-2-phenylindole; DG, dentate gyrus.
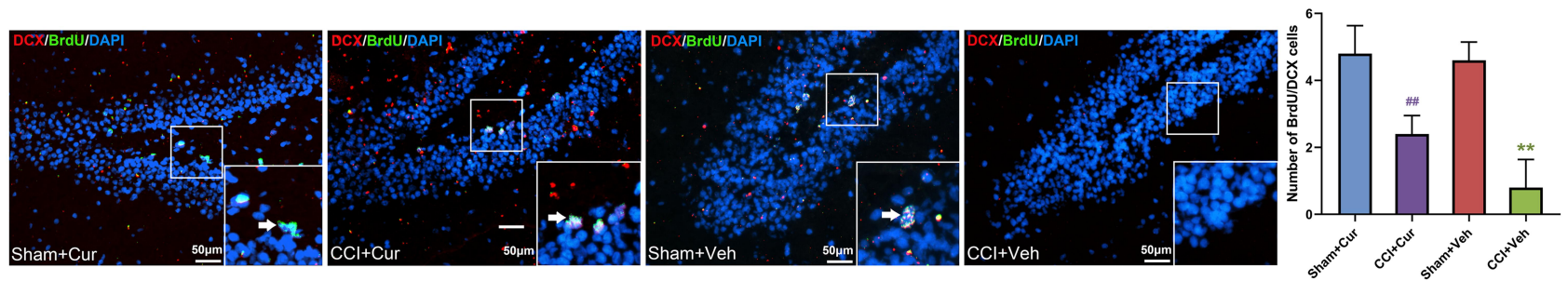

Figure 6 Representative images and quantitative summary of hippocampal DCX/BrdU cells in the DG region from rats in different groups (The white arrows represent $\mathrm{DCX} / \mathrm{BrdU}$ double labeled neurons).

Note: $* * \mathrm{P}<0.05$ vs Sham+Veh, \# $\mathrm{P}<0.05$ vs $\mathrm{CCl}+\mathrm{Veh}$.

Abbreviations: $\mathrm{CCl}$, chronic constriction injury; Veh, vehicle; Cur, curcumin; BrdU, bromodeoxyuridine; DCX, doublecortin; DAPI, 4',6-diamidino-2-phenylindole; DG, dentate gyrus.

had reported that the enhancement effects of curcumin on hippocampal neurogenesis might attribute to the activation of the Extracellular Signal Regulated Kinase (ERK) and Mitogen Activated Protein (MAP) kinase pathways. ${ }^{29}$ More recently, the promotion effect of curcumin treatment on neurogenesis and memory was found to be via activation of adenosine monophosphate-activated protein kinase (AMPK)/c-Jun N-terminal kinase (JNK) signaling, which mediated both mammalian target of rapamycin (mTOR) inhibition and B-cell lymphoma-2 (Bcl-2) upregulation and in turn respectively enhanced autophagy and suppressed apoptosis in hippocampus..$^{30,31}$ 

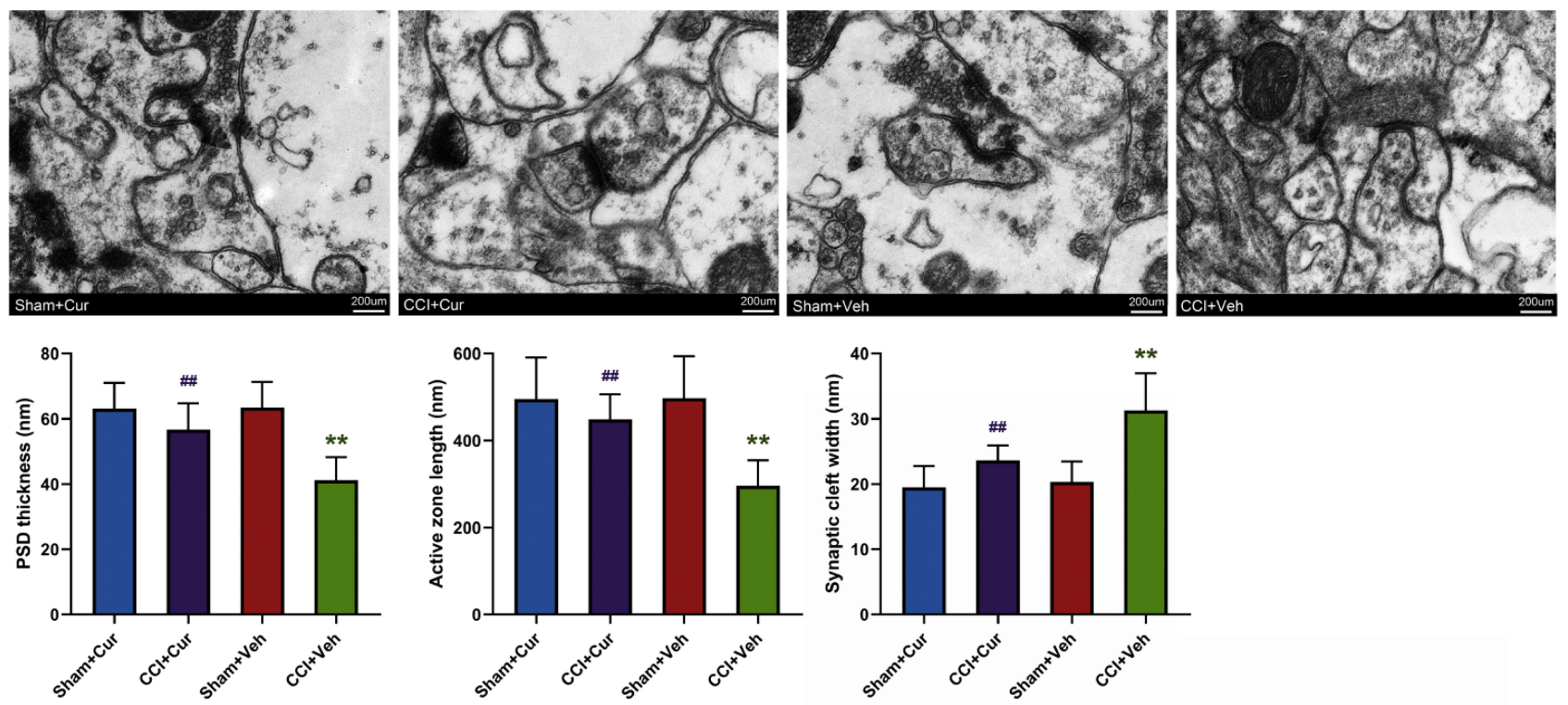

Figure 7 Representative images and quantitative summary of synaptic ultrastructure in the hippocampal DG region as visualized by TEM. Note: ${ }^{* * P}<0.05$ vs Sham+Veh, \#\# $<0.05$ vs $\mathrm{CCl}+\mathrm{Veh}$.

Abbreviations: $\mathrm{CCl}$, chronic constriction injury; Veh, vehicle; Cur, curcumin; PSD, postsynaptic dense; DG, dentate gyrus; TEM, transmission electron microscopy.

More importantly, production of new neurons in the hippocampus alone does not represent the complete process of neurogenesis, because the newly generated neurons must experience differentiation, maturation and then involve in formation of synapses. ${ }^{32}$ In this study, the curcumin-treatment increased not only newly born immature (BrdU/NeuN) cell number but also newly generated mature neurons (BrdU/ DCX) number, simultaneously. Moreover, the PSD thickness and shorter active zone length in parallel with narrow of synaptic cleft width returned to be regular in hippocampus tissue of CCI rats along with curcumin administration. Consequently, these current results indicated the possible involvement of neuronal and synaptic plasticities in the beneficial effect of curcumin on neuropathic pain-induced cognitive impairment.

Besides pro-neurogenic effect, the anti-inflammatory and anti-oxidant effects of curcumin on neuropathic pain had also been demonstrated by previous studies. It was reported that the administration of curcumin can ameliorate pain-related behavior and downregulate the production of pro-inflammatory cytokines, such as IL-1 $\beta$, partly by inhibiting the aggregation of NALP1 inflammasome and the activation of the JAK2STAT3 cascade in astrocytes. ${ }^{33}$ In the oxaliplatin-induced peripheral neuropathic pain and diabetic neuropathy, curcumin could not only inhibit the activation of NF- $\mathrm{kB}$ and inflammatory factors, but inhibiting the level of oxidative stress as well. ${ }^{33,34}$ These potential issues of curcumin effects on neuropathic pain warrant further experiments in further research.

\section{Conclusion}

In conclusion, our findings suggest that consecutively administrating $60 \mathrm{mg} / \mathrm{kg}$ curcumin for 14 days alleviates CCI induced nociceptive behaviors and memory deficit. This effect may be associated with hippocampal neurogenesis and synaptic plasticity improvements. Thus, we infer that curcumin would serve as a potential strategy for the cognitive impairment restoration under prolonged neuropathic pain condition. However, further clinical studies will be performed to clarify this possibility.

\section{Funding}

This work was supported by the National Natural Science Foundation of China [grant number 81701104], the Fundamental Research Funds for the Central Universities (20ykpy25) and the Sun Yat-sen University Clinical Research 5010 Program (2017016).

\section{Disclosure}

The authors report no conflicts of interest in this work.

\section{References}

1. Xia SH, Hu SW, Ge DG, et al. Chronic Pain Impairs Memory Formation via Disruption of Neurogenesis Mediated by Mesohippocampal Brain-Derived Neurotrophic Factor Signaling. Biol Psychiatry. 2020;88(8):597-610. doi:10.1016/j.biopsych.2020.02.013

2. Mazza S, Frot M, Rey AE. Rey AE: a comprehensive literature review of chronic pain and memory. Prog Neuropsychopharmacol Biol Psychiatry. 2018;87(Pt B):183-192. doi:10.1016/j.pnpbp.2017.08.006 
3. McCracken LM, Iverson GL. Iverson GL: predicting complaints of impaired cognitive functioning in patients with chronic pain. $J$ Pain Symptom Manage. 2001;21(5):392-396. doi:10.1016/S08853924(01)00267-6

4. Povedano M, Gascon J, Galvez R, Ruiz M, Rejas J. Rejas J: cognitive function impairment in patients with neuropathic pain under standard conditions of care. J Pain Symptom Manage. 2007;33(1):78-89. doi:10.1016/j.jpainsymman.2006.07.012

5. Xiong B, Zhang W, Zhang L, et al. Tian X: hippocampal glutamatergic synapses impairment mediated novel-object recognition dysfunction in rats with neuropathic pain. Pain. 2020;161(8):1824-1836. doi:10.1097/j.pain.0000000000001878

6. Saffarpour S, Nasirinezhad F. The CA1 hippocampal serotonin alterations involved in anxiety-like behavior induced by sciatic nerve injury in rats. Scand J Pain. 2020;21(1):135-144. doi:10.1515/ sjpain-2020-0037

7. Saffarpour S, Janzadeh A, Rahimi B, Ramezani F, Nasirinezhad F. Chronic nanocurcumin treatment ameliorates pain-related behavior, improves spatial memory, and reduces hippocampal levels of IL-1 $\beta$ and TNF $\alpha$ in the chronic constriction injury model of neuropathic pain. Psychopharmacology. 2021;238(3):877-886. doi:10.1007/ s00213-020-05739-x

8. Apkarian AV, Mutso AA, Centeno MV, et al. Role of adult hippocampal neurogenesis in persistent pain. Pain. 2016;157(2):418-428. doi:10.1097/j.pain.0000000000000332

9. Gao C. Shen J: metabolic Factors and Adult Neurogenesis: impacts of Chinese Herbal Medicine on Brain Repair in Neurological Diseases. Int Rev Neurobiol. 2017;135:117-147.

10. Dellarole A, Morton P, Brambilla R, et al. Bethea JR: neuropathic pain-induced depressive-like behavior and hippocampal neurogenesis and plasticity are dependent on TNFR1 signaling. Brain Behav Immun. 2014;41:65-81. doi:10.1016/j.bbi.2014.04.003

11. Dimitrov EL, Tsuda MC, Cameron HA, Usdin TB. Usdin TB: anxiety- and depression-like behavior and impaired neurogenesis evoked by peripheral neuropathy persist following resolution of prolonged tactile hypersensitivity. $J$ Neurosci. 2014;34 (37):12304-12312. doi:10.1523/JNEUROSCI.0312-14.2014

12. Liu S, Li Q, Zhang MT, et al. Wang YQ: curcumin ameliorates neuropathic pain by down-regulating spinal IL-1beta via suppressing astroglial NALP1 inflammasome and JAK2-STAT3 signalling. Sci Rep. 2016;6:28956. doi:10.1038/srep28956

13. Zhu X, Li Q, Chang R, Yang D, Song Z, Guo Q. Huang C: curcumin alleviates neuropathic pain by inhibiting p300/CBP histone acetyltransferase activity-regulated expression of BDNF and cox-2 in a rat model. PLoS One. 2014;9(3):e91303. doi:10.1371/journal. pone. 0091303

14. Sun J, Chen F, Braun C, et al. Ye DW: role of curcumin in the management of pathological pain. Phytomedicine. 2018;48:129-140. doi:10.1016/j.phymed.2018.04.045

15. Arora R, Kuhad A, Kaur IP, Chopra K. Curcumin loaded solid lipid nanoparticles ameliorate adjuvant-induced arthritis in rats. Eur J Pain. 2015;19(7):940-952. doi:10.1002/ejp.620

16. Zhang L, Ding X, Wu Z, Wang M, Tian M. Tian M: curcumin alleviates pain and improves cognitive impairment in a rat model of cobra venom-induced trigeminal neuralgia. $J$ Pain Res. 2018;11:1095-1104. doi:10.2147/JPR.S162668

17. Bennett GJ, Xie Y-K. Xie YK: a peripheral mononeuropathy in rat that produces disorders of pain sensation like those seen in man. Pain. 1988;33(1):87-107. doi:10.1016/0304-3959(88)90209-6

18. Dai WL, Yan B, Bao YN, Fan JF. Liu JH: suppression of peripheral NGF attenuates neuropathic pain induced by chronic constriction injury through the TAK1-MAPK/NF-kappaB signaling pathways. Cell Commun Signal. 2020;18(1):66. doi:10.1186/s12964-02000556-3
19. Li Y, Jiao H, Ren W, Ren F. Ren F: TRESK alleviates trigeminal neuralgia induced by infraorbital nerve chronic constriction injury in rats. Mol Pain. 2019;15:1744806919882511. doi:10.1177/ 1744806919882511

20. Hossain MM, Belkadi A, Al-Haddad S, Richardson JR. Richardson JR: deltamethrin exposure inhibits adult hippocampal neurogenesis and causes deficits in learning and memory in mice. Toxicol Sci. 2020;178(2):347-357. doi:10.1093/toxsci/kfaa144

21. Chen X, Zhou X, Yang L, et al. Neonatal Exposure to Low-Dose (1.2\%) Sevoflurane Increases Rats' Hippocampal Neurogenesis and Synaptic Plasticity in Later Life. Neurotox Res. 2018;34(2):188-197. doi:10.1007/s12640-018-9877-3

22. Yan Y, Cheng L, Chen X, et al. Ai J: estrogen deficiency is associated with hippocampal morphological remodeling of early postmenopausal mice. Oncotarget. 2017;8(13):21892-21902. doi:10.18632/ oncotarget. 15702

23. Wojtowicz JM, Kee N. Kee N: brdU assay for neurogenesis in rodents. Nat Protoc. 2006;1(3):1399-1405. doi:10.1038/ nprot.2006.224

24. Kempermann G, Jessberger S, Steiner B, Kronenberg G. Kronenberg $\mathrm{G}$ : milestones of neuronal development in the adult hippocampus. Trends Neurosci. 2004;27(8):447-452. doi:10.1016/j. tins.2004.05.013

25. Saffarpour S, Shaabani M, Naghdi N, Farahmandfar M, Janzadeh A, Nasirinezhad F. In vivo evaluation of the hippocampal glutamate, GABA and the BDNF levels associated with spatial memory performance in a rodent model of neuropathic pain. Physiol Behav. 2017;175:97-103. doi:10.1016/j.physbeh.2017.03.025

26. van Praag H, Schinder AF, Christie BR, Toni N, Palmer TD, Gage FH. Gage FH: functional neurogenesis in the adult hippocampus. Nature. 2002;415(6875):1030-1034. doi:10.1038/4151030a

27. Nakashiba T, Cushman JD, Pelkey KA, et al. Young dentate granule cells mediate pattern separation, whereas old granule cells facilitate pattern completion. Cell. 2012;149(1):188-201. doi:10.1016/j. cell.2012.01.046

28. Deng W, Aimone JB, Gage FH. Gage FH: new neurons and new memories: how does adult hippocampal neurogenesis affect learning and memory? Nat Rev Neurosci. 2010;11(5):339-350. doi:10.1038/ nrn2822

29. Kim SJ, Son TG, Park HR, et al. Lee J: curcumin stimulates proliferation of embryonic neural progenitor cells and neurogenesis in the adult hippocampus. $J$ Biol Chem. 2008;283(21):14497-14505. doi:10.1074/jbc.M708373200

30. Yi LT, Dong SQ, Wang SS, et al. Cheng J: curcumin attenuates cognitive impairment by enhancing autophagy in chemotherapy. Neurobiol Dis. 2020;136:104715. doi:10.1016/j.nbd.2019.104715

31. Namgyal D, Ali S, Mehta R, Sarwat M. Sarwat M: the neuroprotective effect of curcumin against Cd-induced neurotoxicity and hippocampal neurogenesis promotion through CREB-BDNF signaling pathway. Toxicology. 2020;442:152542. doi:10.1016/j. tox.2020.152542

32. Liu S, Li Q, Zhang MT, et al. Wang YQ: curcumin ameliorates neuropathic pain by down-regulating spinal IL-1 $\beta$ via suppressing astroglial NALP1 inflammasome and JAK2-STAT3 signalling. Sci Rep. 2016;6:28956.

33. Zhang X, Guan Z, Wang X, et al. Yue X: curcumin Alleviates Oxaliplatin-Induced Peripheral Neuropathic Pain through Inhibiting Oxidative Stress-Mediated Activation of NF- $\mathrm{kB}$ and Mitigating Inflammation. Biol Pharm Bull. 2020;43(2):348-355. doi:10.1248/ bpb.b19-00862

34. Zhao WC, Zhang B, Liao MJ, et al. Curcumin ameliorated diabetic neuropathy partially by inhibition of NADPH oxidase mediating oxidative stress in the spinal cord. Neurosci Lett. 2014;560:81-85. doi:10.1016/j.neulet.2013.12.019 


\section{Publish your work in this journal}

The Journal of Pain Research is an international, peer reviewed, open access, online journal that welcomes laboratory and clinical findings in the fields of pain research and the prevention and management of pain. Original research, reviews, symposium reports, hypothesis formation and commentaries are all considered for publication. The manuscript management system is completely online and includes a very quick and fair peer-review system, which is all easy to use. Visit http:// www.dovepress.com/testimonials.php to read real quotes from published authors. 